\title{
A New Flexible Bathtub-Shaped Modification of the Weibull Model: Properties and Applications
}

\author{
Qinghu Liao (D), ${ }^{1}$ Zubair Ahmad ${ }^{D},{ }^{2}$ Eisa Mahmoudi $\left(\mathbb{D},{ }^{2}\right.$ and G. G. Hamedani ${ }^{3}$ \\ ${ }^{1}$ School of Public Management, Tianjin University of Commerce, Tianjin, China \\ ${ }^{2}$ Department of Statistics, Yazd University, P. O. Box 89175-741, Yazd, Iran \\ ${ }^{3}$ Department of Mathematical and Statistical Sciences, Marquette University, Milwaukee, WI 53201-1881, USA \\ Correspondence should be addressed to Qinghu Liao; qinghu_liao@outlook.com and Zubair Ahmad; z.ferry21@gmail.com
}

Received 17 January 2020; Revised 7 March 2020; Accepted 23 April 2020; Published 20 May 2020

Academic Editor: Qing Chang

Copyright ( 92020 Qinghu Liao et al. This is an open access article distributed under the Creative Commons Attribution License, which permits unrestricted use, distribution, and reproduction in any medium, provided the original work is properly cited.

Many studies have suggested the modifications and generalizations of the Weibull distribution to model the nonmonotone hazards. In this paper, we combine the logarithms of two cumulative hazard rate functions and propose a new modified form of the Weibull distribution. The newly proposed distribution may be called a new flexible extended Weibull distribution. Corresponding hazard rate function of the proposed distribution shows flexible (monotone and nonmonotone) shapes. Three different characterizations along with some mathematical properties are provided. We also consider the maximum likelihood estimation procedure to estimate the model parameters. For the illustrative purposes, two real applications from reliability engineering with bathtub-shaped hazard functions are analyzed. The practical applications show that the proposed model provides better fits than the other nonnested models.

\section{Introduction}

The hazard rate function (also known as failure rate function) is an important reliability characteristic. It deals with the failure of the system at the time, say $t$, given that the system has not failed prior to time $t$. Among the hazard rate functions, the bathtub-shaped hazard rate curve is well known in reliability engineering. It represents the failure behavior of various engineering systems having initially a decreasing failure rate during the very first phase, a relatively constant failure rate in the middle part of the life (usually called useful life period), and finally an increasing failure rate in the last phase. In the context of the reliability theory, these three phases are known, respectively, as burning, random, and wear-out failure regions.

In the last two decades, many new life distributions capable of modeling data with the bathtub-hazard rate function have been introduced in the literature. Most of them are the modifications and extensions of the two-parameter Weibull distribution. For example, a three-parameter exponentiated Weibull (EW) by Mudholkar and
Srivastava [1] has a bathtub-shaped hazard function. A three-parameter modified Weibull extension (MWEx) by Xie et al. [2] exhibits data modeling with a bathtub shape. The truncated Weibull distribution by Zhang and Xie [3] has a bathtub-shaped hazard function. The two-parameter flexible Weibull extension (FWEx) by Bebbington et al. [4] has increasing, decreasing, or bathtub-shaped. A three-parameter generalization of the Weibull model proposed by Ahmad [5] and a new interesting extension of the Weibull model called Zubair-Weibull (ZW) distribution proposed by Ahmad [5] are capable of modeling the data exhibiting the bathtub-shaped failure rate. A three-parameter extended alpha power transformed Weibull (EAPTW) by Ahmad et al. [6] and a three-parameter new extended alpha power transformed Weibull (NEAPTW) by Ahmad et al. [7] are all having failure rate function that can be increasing, decreasing, or bathtub-shaped.

Lai et al. [8] proposed a new modification of the Weibull distribution called modified Weibull (MW) by multiplying $e^{\lambda x}$ with the cumulative hazard rate function (CHRF) of the Weibull model given by 


$$
F(x)=1-e^{-\beta x^{\alpha} e^{\lambda x}}, \quad x \geq 0, \alpha, \beta, \lambda>0 .
$$

Recent studies of the modified Weibull include betamodified Weibull (BMW) by Silva et al. [9]; Bayes analysis of MW by Upadhyay and Gupta [10]; and Bayes analysis of MW using the MCMC approach [11]. A four-parameter Additive Weibull (AW) with bathtub-shaped hazard rate function consisting of two 2-parameter Weibull distributions proposed by Xie and Lai [12] is given by

$$
F(x)=1-e^{-\beta x^{\alpha}-\gamma x^{\theta}}, \quad x \geq 0, \alpha, \beta, \gamma, \theta>0 .
$$

A five-parameter new modified Weibull (NMW) by Almalki and Yuan [13] has cumulative distribution function (CDF) given by

$$
F(x)=1-e^{-\beta x^{\alpha}-\gamma x^{\theta} e^{\lambda \xi}}, \quad x \geq 0, \alpha, \beta, \gamma, \theta, \lambda>0,
$$

which has a bathtub-shaped hazard function consisting of the modified Weibull and Weibull hazards.

Recently, Singh [14] proposed a new model having a bathtub-shaped hazard rate, called the additive Perks-Weibull (APW) by combining the hazard functions of the Perks and Weibull distributions. The CDF of the APW is given by

$$
F(x)=1-\frac{(1+\alpha)}{1+\alpha e^{\lambda x}} e^{-\gamma x^{\theta}}, \quad x \geq 0, \alpha, \theta, \lambda, \gamma>0 .
$$

The key goal of the modification and extension forms of the Weibull model is to describe and fit the data sets with a nonmonotonic hazard rate, such as the bathtub, unimodal, and modified unimodal hazard rate. Many extensions of the Weibull distribution have achieved the above purpose. However, while the number of parameters has increased up to 5 or more, the forms of the survival and hazard functions become complicated and the parameter estimation will be more difficult. On the other hand, unfortunately, some of the modifications do not have a closed form for their CDFs. Furthermore, as we have seen, the bathtub and the modified unimodal shapes have three phases: initially decreasing phase, relatively constant phase, and then an increasing phase for the bathtub shape and the phases of the modified unimodal shape are initially increasing, then decreasing, and then increasing again. The main weakness of some of the modified Weibull distributions is that they are unable to fit the last phase of the bathtub shape.

Due to importance of the statistical distributions in reliability engineering and other related fields, researchers have shown a serious interest to propose new distributions. The new developments have been made through a number of approaches. One approach is to consider a convex combination of two survival functions and generate a new function as

$$
S(x)=\eta_{1} S_{1}(x)+\left(1-\eta_{2}\right) S_{2}(x) \text {, }
$$

where $0<\eta_{1}, \eta_{2}<1$ and $\eta_{1}+\eta_{2}=1$. This approach of introducing new functions is named as a mixture of distributions. One may also generate a new function by looking at a linear combination with two cumulative hazard rate functions as

$$
H(x)=\beta H_{1}(x)+\sigma H_{2}(x) .
$$

In terms of $\mathrm{CHRF}$, the $\mathrm{CDF}$ can be expressed as

$$
G(x)=1-e^{-H(x)}
$$

where the CHRF, denoted by $H(x)$, satisfies the following conditions:

(1) $H(x)$ is a differentiable nonnegative and increasing function of $x$,

(2) $\lim _{x \longrightarrow 0} H(x) \longrightarrow 0$ and $\lim _{x \longrightarrow \infty} H(x) \longrightarrow \infty$.

The probability density function (PDF) corresponding to (7) is given by

$$
g(x)=h(x) e^{-H(x)} .
$$

The modified Weibull extensions proposed by Xie and Lai [12]; Sarhan and Zaindin [15]; Almalki and Yuan [13]; and Lemonte et al. [16] belong to the class (7) with bounded $H(x)$. In this article, however, a new function $\log H(x)$ replaces $H(x)$ to relax the boundary conditions. Hence, the expression (6) can be written as

$$
H(x)=H_{1}^{\beta}(x) \times H_{2}^{\sigma}(x) .
$$

The modified Weibull distributions suggested by Xie et al. [2]; Lai et al. [8]; Nadarajah and Kotz [17]; and Bebbington et al. [4] belong to the class (9). The expression (9) can be expressed as

$$
\log H(x)=\beta \log H_{1}(x)+\sigma \log H_{2}(x) .
$$

Here, a mixture of the two logarithms of cumulative hazard rate functions, taken as $\log H_{1}(x)=x^{\alpha}$ and $\log H_{2}(x)=$ $\left\{-\left(1 / x^{\lambda}\right)\right\}$, is used to introduce a new model called, new flexible extended Weibull (NFEW) distribution. So, the expression (10) can be written as

$$
H(x)=e^{\beta x^{\alpha}-\left(\sigma / x^{\lambda}\right)} .
$$

Using (11) in (7), one arrives at the CDF of the NFEW distribution. The new model is capable of modeling lifetime data with unimodal, modified unimodal, or most importantly with bathtub-shaped failure rates.

The rest of the paper is organized as follows: Section 2 provides the definition and sketch of the proposed distribution. Some basic mathematical properties are derived in Section 3. Section 4 offers the estimation of the model parameters. Certain characterizations of the proposed distribution are discussed in Section 5. Two real-life applications are presented in Section 6. Finally, some concluding remarks are provided in Section 7.

\section{New Flexible Extended Weibull Distribution}

The CDF of the NFEW distribution is given by

$$
\left.F(x)=1-\exp \left\{-e^{\left(\beta x^{\alpha}-\left(\sigma / x^{\lambda}\right)\right.}\right)\right\}, \quad x \geq 0, \alpha, \beta, \sigma, \lambda>0 .
$$
(12) is 


$$
\begin{aligned}
f(x)= & \left(\alpha \beta x^{\alpha-1}+\frac{\lambda \sigma}{x^{\lambda+1}}\right) e^{\left(\beta x^{\alpha}-\left(\sigma / x^{\lambda}\right)\right)} \\
& \cdot \exp \left\{-e^{\left(\beta x^{\alpha}-\left(\sigma / x^{\lambda}\right)\right)}\right\}, \quad x>0 .
\end{aligned}
$$

The survival function (SF) and hazard rate function (HRF) of the proposed model are given, respectively, by

$$
\begin{aligned}
& S(x)=\exp \left\{-e^{\left(\beta x^{\alpha}-\left(\sigma / x^{\lambda}\right)\right)}\right\}, \quad x>0, \\
& h(x)=\left(\alpha \beta x^{\alpha-1}+\frac{\lambda \sigma}{x^{\lambda+1}}\right) e^{\left(\beta x^{\alpha}-\left(\sigma / x^{\lambda}\right)\right)}, \quad x>0 .
\end{aligned}
$$

Some possible shapes for the PDF of the proposed model are shown in Figure 1.

Some possible shapes for the HRF of the proposed model are sketched in Figures 2 and 3.

2.1. Motivations. The key motivations for using the proposed model in practice are the following:

(1) The distribution function as well as the survival function of the proposed model have closed forms.

(2) It is capable of modeling data with monotonic and nonmonotonic failure rates.

(3) The proposed model is capable of modeling the last phase of the modified unimodal-shaped failure rate function closely (see Figure 2).

(4) It has a long constant failure rate period (as shown in Figure 3) which is capable to model the second phase of the bathtub-shaped failure rate.

(5) It is capable of modeling the last phase of the bathtub-shaped failure rate closely (see Figure 3).

(6) It may provide a better fit to the reliability data having a bathtub-shaped failure rate function than the other well-known bathtub-shaped extensions of the Weibull distribution with the same and higher number of parameters.

\section{Basic Properties}

In this section, some basic properties of the proposed model are derived.

3.1. Quantile and Median. The expression for the qth quantile, say $x_{q}$, of the NFEW model is given by

$$
\beta x_{q}^{\alpha}-\frac{\sigma}{x_{q}^{\lambda}}-\log \{-\log (1-q)\}=0 .
$$

Using $q=0.5$ in (15), one obtains the median of the NFEW model. Also, setting $q=0.25$ and $q=0.75$ in (15), one obtains the 1st and the3rd quartiles of the NFEW distribution, respectively.

3.2. Generation of Random Numbers. The expression for generating random numbers from NFEW distribution is given by

$$
\beta x^{\alpha}-\frac{\sigma}{x^{\lambda}}-\log \{-\log (1-R)\}=0, \quad R \sim U(0,1) .
$$

3.3. Moments. Generally speaking, we always need to keep in mind the importance of the moments in any statistical analysis particularly in applied fields. For example, through moments, the important characteristics such as tendency, dispersion, skewness, and kurtosis of a distribution can be studied. If $X$ has the NFEW distribution with parameters vector $(\alpha, \beta, \sigma, \lambda)$, the $r$ th moment of $X$ is derived as

$$
\mu_{r}^{\prime}=\int_{0}^{\infty} x^{r} f(x) \mathrm{d} x .
$$

Using (13) in (17), we obtain

$$
\mu_{r}^{\prime}=\sum_{i=0}^{\infty} \sum_{j=0}^{\infty} \frac{(-1)^{i}(i+1)^{j} \beta^{j}}{i ! j !}\left\{\alpha \beta \int_{0}^{\infty} x^{r+\alpha(j+1)-1} e^{-\left\{\sigma(i+1) / x^{\lambda}\right\}} \mathrm{d} x+\lambda \sigma \int_{0}^{\infty} x^{r+\alpha j-\lambda-1} e^{-\left\{\sigma(i+1) / x^{\lambda}\right\}} \mathrm{d} x\right\} .
$$

Finally, the following expression is observed:

$$
\begin{aligned}
\mu_{r}^{\prime}= & \sum_{i=0}^{\infty} \sum_{j=0}^{\infty} \frac{(-1)^{i}(i+1)^{j} \beta^{j}}{i ! j !} \\
& \cdot\left\{\alpha \beta \lambda \frac{\Gamma(r+\alpha(j+1)-2 / \lambda)}{\sigma(i+1)^{r+\alpha(j+1)-2 / \lambda}}+\lambda^{2} \sigma \frac{\Gamma(r+\alpha j-(\lambda-1)-2 / \lambda)}{\sigma(i+1)^{r+\alpha j-(\lambda-1)-2 / \lambda}}\right\} .
\end{aligned}
$$

3.4. hth Order Negative Moments. The hth order negative moment of the NFEW random variable $X$ is derived as

$$
\begin{aligned}
& \mu_{-h}^{\prime}=\int_{0}^{\infty} x^{-h} f(x) \mathrm{d} x, \\
& \mu_{-h}^{\prime}=\int_{0}^{\infty} x^{r}\left(\alpha \beta x^{\alpha-1}+\frac{\lambda \sigma}{x^{\lambda+1}}\right) e^{\left(\beta x^{\alpha}-\left(\sigma / x^{\lambda}\right)\right)} \exp \left\{-e^{\left(\beta x^{\alpha}-\left(\sigma / x^{\lambda}\right)\right)}\right\} \mathrm{d} x .
\end{aligned}
$$




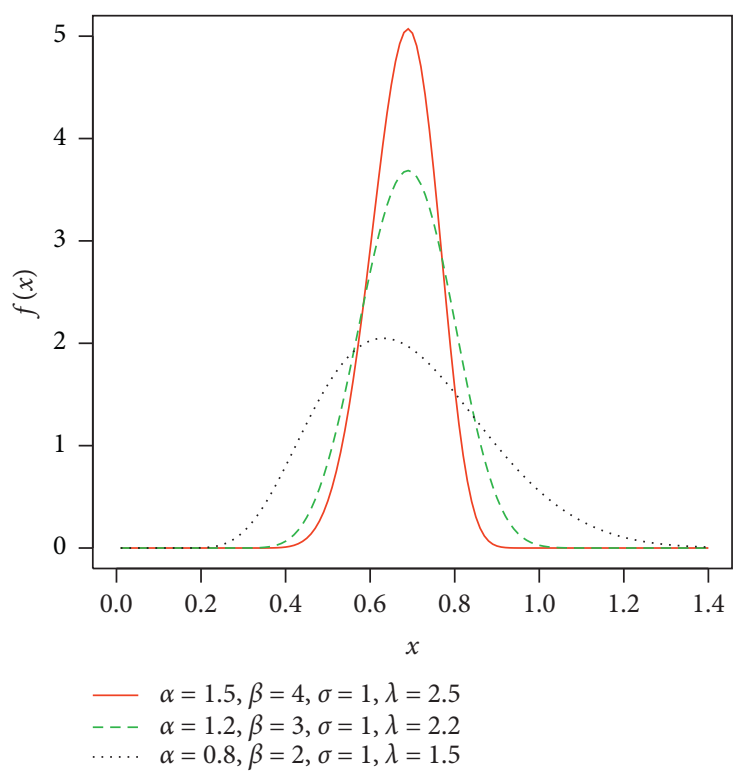

(a)

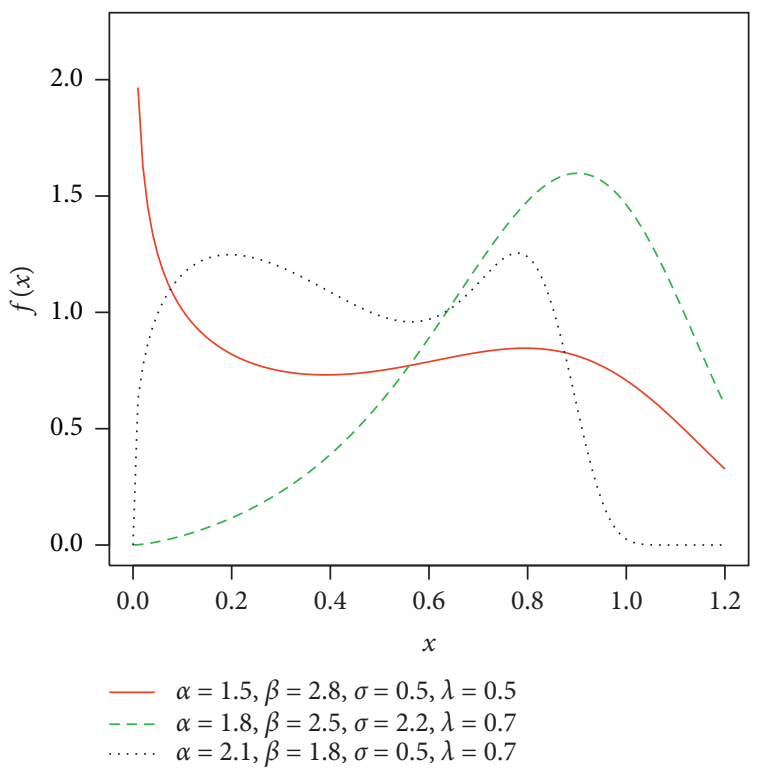

(b)

Figure 1: Plots of PDF of the NFEW distribution for selected parameter values.

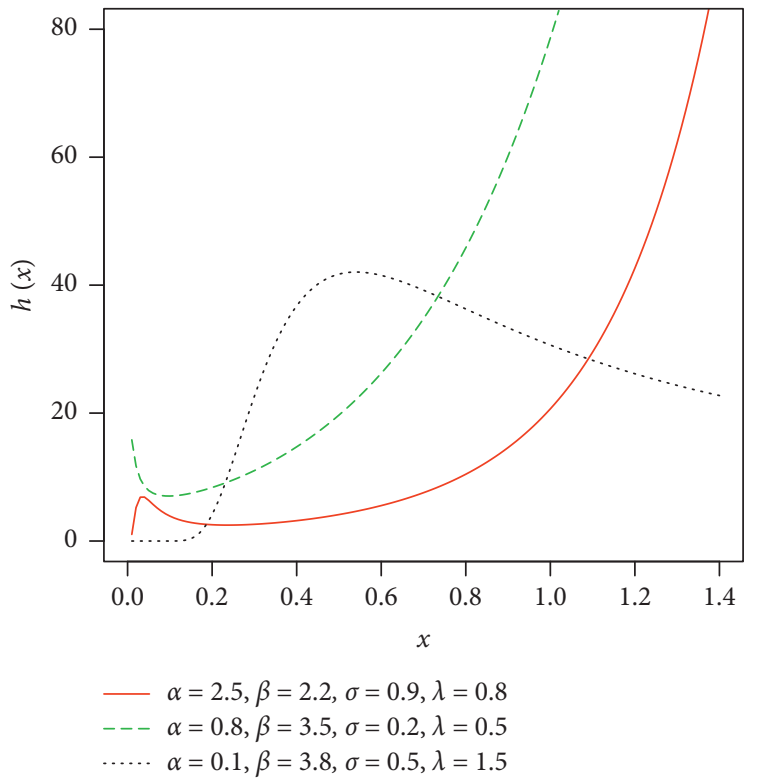

(a)

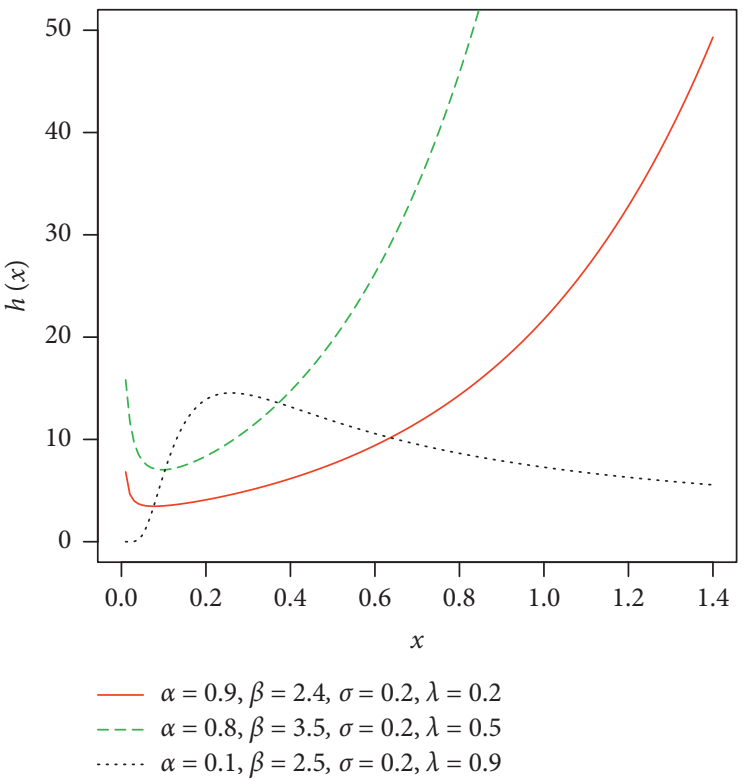

(b)

Figure 2: Plots of HRF of the NFEW distribution for selected values of parameters.

We have the following expression:

$$
\begin{aligned}
\mu_{-h}^{\prime}= & \sum_{i=0}^{\infty} \sum_{j=0}^{\infty} \frac{(-1)^{i}(i+1)^{j} \beta^{j}}{i ! j !} \\
& \cdot\left\{\alpha \beta \lambda \frac{\Gamma(\alpha(j+1)-h-2 / \lambda)}{\sigma(i+1)^{\alpha(j+1)-h-2 / \lambda}}\right. \\
& \left.+\lambda^{2} \sigma \frac{\Gamma(\alpha j-(\lambda-1)-h-2 / \lambda)}{\sigma(i+1)^{\alpha j-(\lambda-1)-h-2 / \lambda}}\right\} .
\end{aligned}
$$

3.5. Order Statistics. Let $X_{1}, X_{2}, \ldots, X_{k}$ be a random sample from NFEW distribution with parameters $(\alpha, \beta, \sigma, \lambda)$ and let $X_{(1: k)} \leq \ldots \leq X_{(k: k)}$ be the corresponding order statistics. The density function of $X_{(r: k)}$ for $r=1,2,3, \ldots, k$ is given by

$$
f_{r: k}(x)=\frac{f(x)}{B(r, k-r+1)} \sum_{v=0}^{k-r}\left(\begin{array}{c}
k-r \\
v
\end{array}\right)(-1)^{v} F(x)^{v+r-1},
$$




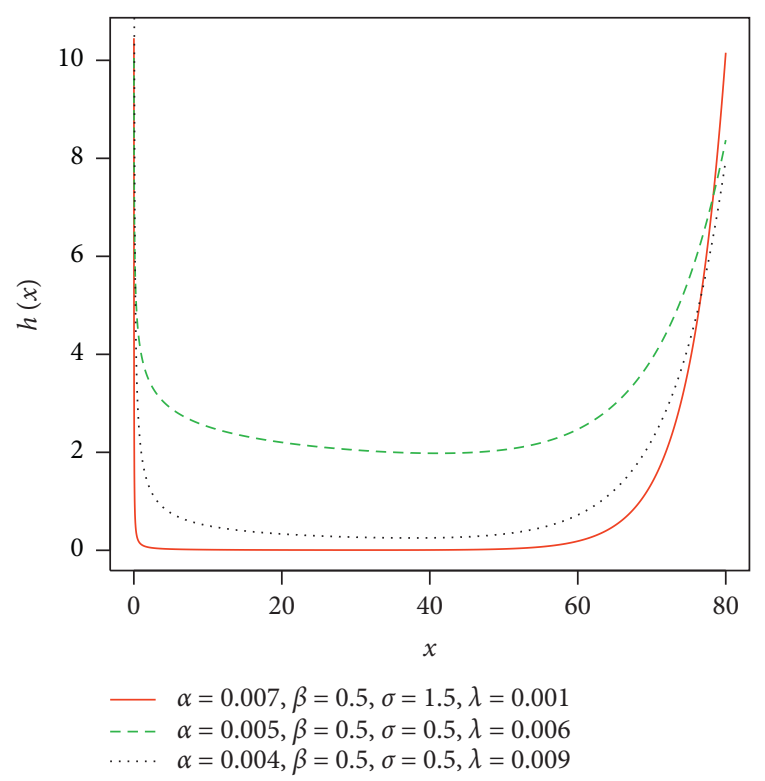

FIGURE 3: Plots of HRF of the NFEW distribution for selected values of parameters.

where $B(.$, .) denotes the beta function. Using (12) and (13) in (22), we obtain the densities of the order statistics of NFEW distribution.

\section{Maximum Likelihood Estimation}

In this section, the maximum likelihood estimates of the model parameters are obtained. Let $x_{1}, x_{2}, \ldots, x_{k}$ be observed values of a random sample from the NFEW distribution with parameters $(\alpha, \beta, \sigma, \lambda)$. Then, the log-likelihood function of this sample is

$$
\begin{aligned}
\ln L= & \sum_{i=1}^{k} \log \left(\alpha \beta x_{i}^{\alpha-1}+\frac{\lambda \sigma}{x_{i}^{\lambda+1}}\right)+\sum_{i=1}^{k}\left(\beta x_{i}^{\alpha}-\frac{\sigma}{x_{i}^{\lambda}}\right) \\
& -\sum_{i=1}^{k} e^{\left(\beta x_{i}^{\alpha}-\left(\sigma / x_{i}^{\lambda}\right)\right)} .
\end{aligned}
$$

Computing the partial derivatives of (23) with respect to the model parameters and then setting the result equal to zero, one has

$$
\begin{aligned}
\frac{\partial}{\partial \alpha} \ln L & =\beta \sum_{i=1}^{k} \frac{\left(\alpha x_{i}^{\alpha-1} \log \left(x_{i}\right)+x_{i}^{\alpha-1}\right)}{\left(\alpha \beta x_{i}^{\alpha-1}+\left(\lambda \sigma / x_{i}^{\lambda+1}\right)\right)}+\beta \sum_{i=1}^{k} x_{i}^{\alpha} \log \left(x_{i}\right)-\beta \sum_{i=1}^{k} \log \left(x_{i}\right) x_{i}^{\alpha} e^{\left(\beta x_{i}^{\alpha}-\left(\sigma / x_{i}^{\lambda}\right)\right)}=0, \\
\frac{\partial}{\partial \beta} \ln L & =\sum_{i=1}^{k} \frac{\alpha x_{i}^{\alpha-1}}{\left(\alpha \beta x_{i}^{\alpha-1}+\left(\lambda \sigma / x_{i}^{\lambda+1}\right)\right)}+\sum_{i=1}^{k} x_{i}^{\alpha}-\sum_{i=1}^{k} x_{i}^{\alpha} e^{\left(\beta x_{i}^{\alpha}-\left(\sigma / x_{i}^{\lambda}\right)\right)}=0, \\
\frac{\partial}{\partial \sigma} \ln L & =\sum_{i=1}^{k} \frac{\lambda / x_{i}^{\lambda+1}}{\left(\alpha \beta x_{i}^{\alpha-1}+\left(\lambda \sigma / x_{i}^{\lambda+1}\right)\right)}-\sum_{i=1}^{k} \frac{1}{x_{i}^{\lambda}}+\sum_{i=1}^{k} \frac{e^{\left(\beta x_{i}^{\alpha}-\left(\sigma / x_{i}^{\lambda}\right)\right)}}{x_{i}^{\lambda}}=0, \\
\frac{\partial}{\partial \lambda} \ln L & =\sum_{i=1}^{k} \frac{\sigma}{\left(\alpha \beta x_{i}^{\alpha-1}+\left(\lambda / x_{i}^{\lambda+1}\right)\right)}\left(\frac{x_{i}^{\lambda+1}-\lambda x_{i}^{\lambda+1} \log \left(x_{i}\right)}{\left(x_{i}^{\lambda+1}\right)^{2}}\right)+\sum_{i=1}^{k} \frac{\log \left(x_{i}\right)}{x_{i}^{\lambda}}-\sigma \sum_{i=1}^{k} \frac{e^{\left(\beta x_{i}^{\alpha}-\left(\sigma / x_{i}^{\lambda}\right)\right)} \log \left(x_{i}\right)}{x_{i}^{\lambda}}=0 .
\end{aligned}
$$

From (24)-(27), it is clear that these expressions do not have closed form solutions. Therefore, the estimates of the model parameters can be obtained numerically by using the iterating procedure. The "SANN" algorithm is used in the R language to obtain the numerical estimates of the model parameters in this paper.

\section{Characterizations}

In this section, we present certain characterizations of NFEW distribution. The first characterization is based on the hazard function, the second one is based on the ratio of two truncated moments, and the third is based on conditional expectation of certain function of the random variable.

5.1. Characterization in terms of Hazard Function. The hazard function $h_{F}(x)$ of a twice differentiable distribution function, $F(x)$, satisfies the following first order differential equation:

$$
\frac{f^{\prime}(x)}{f(x)}=\frac{h_{F}^{\prime}(x)}{h_{F}(x)}-h_{F}(x) .
$$


It should be mentioned that for many univariate continuous distributions, the above equation is the only differential equation available in terms of the hazard function. In this section, we present a characterization of NFEW which is not of the above trivial form.

Proposition 1. Let $X: \Omega \longrightarrow(0, \infty)$ be a continuous random variable. The random variable $X$ has $P D F(13)$ if and only if its hazard function $h_{F}(x)$ satisfies the following differential equation:

$$
\begin{aligned}
& h_{G}^{\prime}(x)-\left(\alpha \beta x^{\alpha-1}+\frac{\lambda \sigma}{x^{\lambda+1}}\right) h_{F}(x) \\
& \quad=\left(\alpha(\alpha-1) \beta x^{\alpha-2}-\frac{\lambda(\lambda+1) \sigma}{x^{\lambda+2}}\right) \exp \left\{\beta x^{\alpha}-\frac{\sigma}{x^{\lambda}}\right\}, \quad x>0 .
\end{aligned}
$$

Proof. If $X$ has PDF (13), then clearly the above differential equation holds. Now, if the differential equation holds, then

$$
\begin{aligned}
& \frac{\mathrm{d}}{\mathrm{d} x}\left[\exp \left\{-\left(\beta x^{\alpha}-\frac{\sigma}{x^{\lambda}}\right)\right\} h_{F}(x)\right] \\
& \quad=\left(\alpha(\alpha-1) \beta x^{\alpha-2}-\frac{\lambda(\lambda+1) \sigma}{x^{\lambda+2}}\right)=\frac{\mathrm{d}}{\mathrm{d} x}\left(\alpha \beta x^{\alpha-1}-\frac{\lambda \sigma}{x^{\lambda+1}}\right), \quad x>0,
\end{aligned}
$$

which gives the hazard function corresponding to the PDF given by (13).

5.2. Characterizations Based on Two Truncated Moments. In this section, we present characterizations of the NFEW distribution in terms of a simple relationship between two truncated moments. Our first characterization employs a theorem due to Glänzel [18], see Theorem 1 of the Appendix. Note that the result holds also when the interval is not closed. Moreover, it could be also applied when the CDF $F(x)$ does not have a closed form. As shown by Glanzel [19], this characterization is stable in the sense of weak convergence.

Proposition 2. Let $X: \Omega \longrightarrow(0, \infty)$ be a continuous random variable and let $q_{1}(x) \equiv 1$ and $q_{2}(x)=\exp [-$ $\left.\left\{\exp \left(\beta x^{\alpha}-\left(\sigma / x^{\lambda}\right)\right)\right\}\right]$ for $x>0$. The random variable $X$ has PDF (13) if and only if the function $\eta(x)$ defined in Theorem 1 has the form

$$
\eta(x)=\frac{1}{2} \exp \left[-\left\{\exp \left(\beta x^{\alpha}-\frac{\sigma}{x^{\lambda}}\right)\right\}\right], \quad x>0 .
$$

Proof. Let $X$ be a random variable with PDF (13), then

$$
\begin{aligned}
& (1-F(x)) E\left(q_{1}(X) \mid X \geq x\right)=\exp \left[-\left\{\exp \left(\beta x^{\alpha}-\frac{\sigma}{x^{\lambda}}\right)\right\}\right], \quad x>0, \\
& (1-F(x)) E\left(q_{2}(X) \mid X \geq x\right)=\frac{1}{2} \exp \left[-2\left\{\exp \left(\beta x^{\alpha}-\frac{\sigma}{x^{\lambda}}\right)\right\}\right], \quad x>0,
\end{aligned}
$$

and finally

$$
\eta(x) q_{1}(x)-q_{2}(x)=\frac{1}{2} \exp \left[-\left\{\exp \left(\beta x^{\alpha}-\frac{\sigma}{x^{\lambda}}\right)\right\}\right]<0, \quad \text { for } x>0 .
$$

Conversely, if $\eta(x)$ is given as above, then

$$
\begin{aligned}
s^{\prime}(x) & =\frac{\eta^{\prime}(x) q_{1}(x)}{\eta(x) q_{1}(x)-q_{2}(x)} \\
& =\left(\alpha \beta x^{\alpha-1}-\frac{\lambda \sigma}{x^{\lambda+1}}\right) \exp \left\{\beta x^{\alpha}-\frac{\sigma}{x^{\lambda}}\right\}, \quad x>0 .
\end{aligned}
$$

Now, in view of Theorem $1, X$ has density (13).

Corollary 1. Let $X: \Omega \longrightarrow(0, \infty)$ be a continuous random variable and let $q_{1}(x)$ be as in Proposition 2. The random variable $X$ has PDF (13) if and only if there exist functions $q_{2}(x)$ and $\eta(x)$ defined in Theorem 1 satisfying the following differential equation:

$$
\frac{\eta^{\prime}(x) q_{1}(x)}{\eta(x) q_{1}(x)-q_{2}(x)}=\left(\alpha \beta x^{\alpha-1}-\frac{\lambda \sigma}{x^{\lambda+1}}\right) \exp \left\{\beta x^{\alpha}-\frac{\sigma}{x^{\lambda}}\right\}, \quad x>0 .
$$

Corollary 2. The general solution of the differential equation in Corollary 1 is

$$
\begin{aligned}
\eta(x)= & \exp \left\{-\exp \left(\beta x^{\alpha}-\frac{\sigma}{x^{\lambda}}\right)\right\}\left[-\int\left(\alpha \beta x^{\alpha-1}-\frac{\lambda \sigma}{x^{\lambda+1}}\right)\right. \\
& \cdot \exp \left(\beta x^{\alpha}-\frac{\sigma}{x^{\lambda}}\right) \exp \left\{-\exp \left(\beta x^{\alpha}-\frac{\sigma}{x^{\lambda}}\right)\right\} \\
& \left.\times\left(q_{1}(x)\right)^{-1} q_{2}(x) \mathrm{d} x+D\right],
\end{aligned}
$$

where $D$ is a constant. We like to point out that one set of functions satisfying the above differential equation is given in Proposition 2 with $D=0$. Clearly, there are other triplets $\left(q_{1}, q_{2}, \eta\right)$ which satisfy conditions of Theorem 1 .

5.3. Characterization Based on the Conditional Expectation of Certain Function of the Random Variable. In this section, we employ a single function $\psi$ of $X$ and characterize the distribution of $X$ in terms of the truncated moment of $\psi(X)$. The following proposition has already appeared in Hamedani [20], so we will just state it here which can be used to characterize the NFEW distribution.

Proposition 3. Let $X: \Omega \longrightarrow(e, f)$ be a continuous random variable with $\operatorname{CDF} F(x)$. Let $\psi(x)$ be a differentiable function on $(e, f)$ with $\lim _{x \longrightarrow e^{+}} \psi(x)=1$. Then, for $\delta \neq 1$,

$$
E[\psi(X) \mid X \geq x]=\delta \psi(x), \quad x \in(e, f),
$$

if and only if

$$
\psi(x)=\{1-F(x)\}^{(1 / \delta)-1}, \quad x \in(e, f) .
$$


Remark 1. For $(e, f)=(0, \infty), \psi(x)=\exp \left[-\left\{\exp \left(\beta x^{\alpha}-\right.\right.\right.$ $\left.\left.\left.\left(\sigma / x^{\lambda}\right)\right)\right\}\right]$, and $\delta=(1 / 2)$, Proposition 3 provides a characterization of the NFEW distribution.

\section{Applications}

For the practical illustration, the results of applying the NFEW distribution to two well-known data sets with bathtub-shaped failure rates are compared to other modified forms of the Weibull distribution such as the exponentiated Weibull (EW) by Mudholkar and Srivastava [1]; Additive Weibull by Xie and Lai [12]; Marshall-Olkin Weibull (MOW) by Marshall and Olkin [21]; new modified Weibull by Almalki and Yuan [13]; and additive Perks-Weibull by Singh [14]. The EW distribution is one of the most interesting modifications of the Weibull distribution offering data modeling with nonmonotonic failure rate function.

The hazard function of the AW distribution is very interesting, offering data modeling with bathtub-shaped hazard rate function. Since there are two modes of failure (early and degradation) in the data set, Xie and Lai [12] showed that it would be better to consider a model with additive hazard containing one decreasing and one increasing hazard rates for analyzing data having a bathtub shape. Almalki and Yuan [13] showed that the NMW distribution fits these two data sets better than beta-modified Weibull (BMW), AW, and modified Weibull distribution by Sarhan and Zaindin [15]. The MOW model is another prominent extension of the Weibull model which has been used for modeling data in reliability engineering and other related fields. Recently, Singh [14] showed that the APW distribution fits these two data sets better than NMW and AW distributions. follows:

The densities of the competing models are given as

(1) The Additive Weibull distribution by Xie and Lai:

$$
\begin{aligned}
f(x)=\left(\alpha \beta x^{\alpha-1}-\theta \gamma x^{\theta-1}\right) e^{-\beta x^{\alpha}-\gamma x^{\theta}}, \\
x>0, \alpha, \beta, \gamma, \theta>0 .
\end{aligned}
$$

(2) The new modified Weibull by Almalki and Yuan:

$$
\begin{aligned}
f(x)=\left(\alpha \beta x^{\alpha-1}-\gamma x^{\theta-1}(x \lambda+\theta) e^{\lambda x}\right) e^{-\beta x^{\alpha}-\gamma x^{\theta} e^{\lambda x},} \\
x>0, \alpha, \beta, \gamma, \theta, \lambda>0 .
\end{aligned}
$$

(3) The additive Perks-Weibull by Singh:

$$
\begin{array}{r}
f(x)=\left(\frac{\alpha \lambda e^{\lambda x}}{1+\alpha e^{\lambda x}}-\theta \gamma x^{\theta-1}\right) \frac{(1+\alpha)}{1+\alpha e^{\lambda x} e^{-\gamma x^{\theta}},} \\
x>0, \alpha, \gamma, \theta, \lambda>0 .
\end{array}
$$

(4) The exponentiated Weibull by Mudholkar and Srivastava:

$$
\begin{array}{r}
f(x)=\alpha \gamma \beta x^{\alpha-1} e^{-\beta x^{\alpha}}\left(1-e^{-\beta x^{\alpha}}\right)^{\gamma-1}, \\
x>0, \alpha, \beta, \gamma>0 .
\end{array}
$$

(5) The Marshall-Olkin Weibull by Marshall and Olkin:

$$
\begin{aligned}
f(x)=\frac{\alpha \beta \theta x^{\alpha-1} e^{-\beta x^{\alpha}}}{\left(1-(1-\theta)\left(1-e^{-\beta x^{\alpha}}\right)\right)^{2}}, & \\
x & >0, \alpha, \beta, \theta>0 .
\end{aligned}
$$

The analytical measures for model comparison such as the Akaike information criterion (AIC), Bayesian information criterion (BIC), Kolmogorov-Smirnov (KS) statistic, and the corresponding $p$ value are considered. Using these statistical measures, it is shown that the NFEW distribution provides a better fit than the competing models.

6.1. Arset Data. The first data set representing the lifetimes of 51 devices taken from [22] is as $0.1,0.2,1,1,1,1,1,2,3,6$, $7,1,1,12,18,18,18,18,18,21,32,36,40,45,46,47,50,55$, $60,63,63,67,67,67,67,72,75,79,82,82,83,84,84,84,85$, $85,85,85,85,86$, and 86 . Many authors have analyzed this data set, including Mudholkar and Srivastava [1]; Xie and Lai [12]; Lai et al. [8]; Sarhan and Zaindin [15]; and Silva et al. [9]. This data set is known to have a bathtub-shaped hazard rate as shown by the scaled TTT-transform plot (Figure 4). Table 1 provides maximum likelihood estimates (MLEs) of the parameters of the NFEW model and other competing distributions with standard errors in brackets, and the goodness of fit measures are provided in Table 2. From Table 2, it is clear that the proposed model could be chosen as the best model among the fitted models since it has the lowest values of the AIC, BIC, and KS. From Figure 5, it is clear that the CDF of NFEW fits the data well and its survival function follows the Kaplan-Meier estimate closely.

6.2. Meeker and Escobar Data. The second data representing the failure times of a sample of 30 devices taken from Meeker and Escobar [23] are given by 2, 10, 13, 23, 23, 28, 30, 65, 80, $88,106,143,147,181,173,212,245,247,261,266,275,293$, $300,300,300,300,300,300,300$, and 300. These data have a bathtub-shaped hazard function as indicated by the scaled TTT-transform plot (Figure 6). The maximum likelihood estimates of the model parameters with standard errors in brackets are provided in Table 3. Again, the proposed distribution provides a better fit than the other competing distributions, as can be seen from Table 4. From Figure 7, it can easily be detected that the CDF of NFEW fits the data well. 


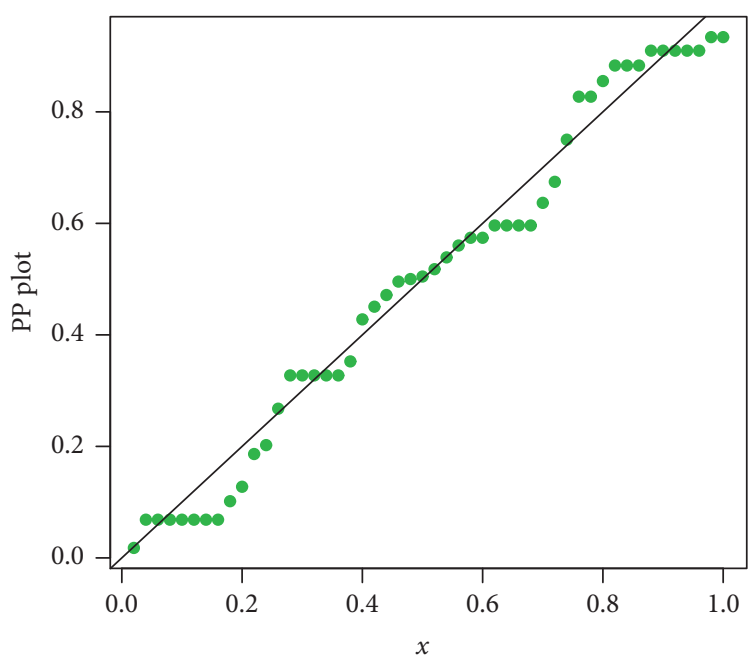

(a)

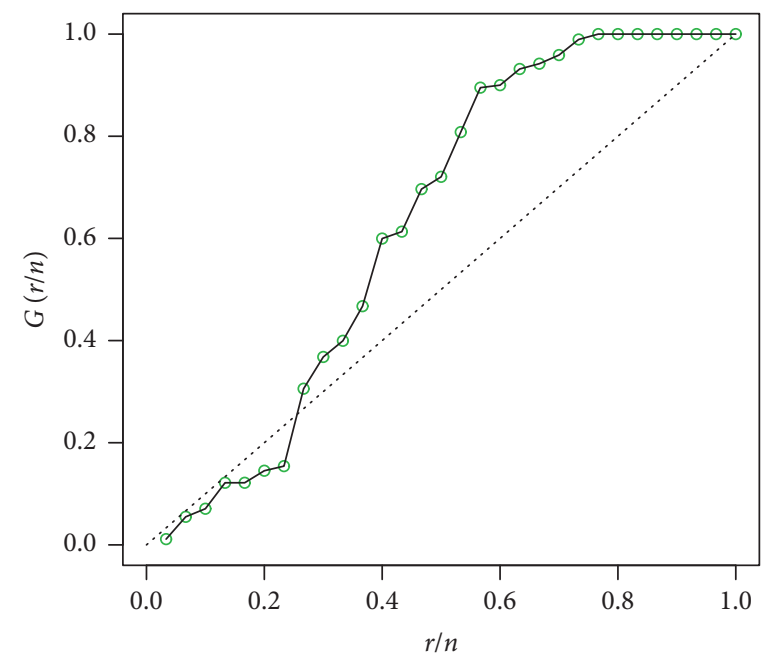

— Data 2

(b)

FIgUre 4: The PP-plot of the NFEW distribution and time scale TTT-transform plot for Arset data.

TABLE 1: MLEs with their standard errors in brackets for the Arset data.

\begin{tabular}{|c|c|c|c|c|c|c|}
\hline Dist. & $\widehat{\beta}$ & $\widehat{\alpha}$ & $\widehat{\gamma}$ & $\widehat{\theta}$ & $\hat{\lambda}$ & $\widehat{\sigma}$ \\
\hline NFEW & $2.011 \times 10^{-5}\left(1.20 \times 10^{-4}\right)$ & $2.133 \times 10^{-9}\left(3.10 \times 10^{-10}\right)$ & & & $0.186(1.008)$ & $0.005(0.003)$ \\
\hline EW & $1.373(0.184)$ & $0.002(0.201)$ & $0.495(0.079)$ & & & \\
\hline MOW & $0.707(0.3698)$ & $0.131(0.259)$ & & $3.620(4.4248)$ & & \\
\hline NMW & $7.01 \times 10^{-8}\left(1.50 \times 10^{-7}\right)$ & $0.071(0.031)$ & $0.016(3.602)$ & $0.595(0.128)$ & $0.197(0.184)$ & \\
\hline AW & $0.086(0.036)$ & $1.13 \times 10^{-8}\left(5.18 \times 10^{-8}\right)$ & $0.102(4.004)$ & $4.214(1.033)$ & & \\
\hline APW & & $7.15 \times 10^{-17}\left(4.36 \times 10^{-8}\right)$ & $0.443(0.005)$ & $0.0532(0.020)$ & $0.6884(0.092)$ & \\
\hline
\end{tabular}

TABLE 2: Summary values of the models fitted to the Arset data.

\begin{tabular}{lcccc}
\hline Dist. & AIC & BIC & KS & $p$ value \\
\hline NFEW & 430.70 & 438.49 & 0.084 & 0.806 \\
EW & 485.97 & 491.77 & 0.201 & 0.036 \\
MOW & 488.88 & 494.68 & 0.178 & 0.076 \\
NMW & 435.86 & 445.48 & 0.088 & 0.803 \\
AW & 451.09 & 458.71 & 0.127 & 0.365 \\
APW & 433.75 & 441.40 & 0.091 & 0.804 \\
\hline
\end{tabular}

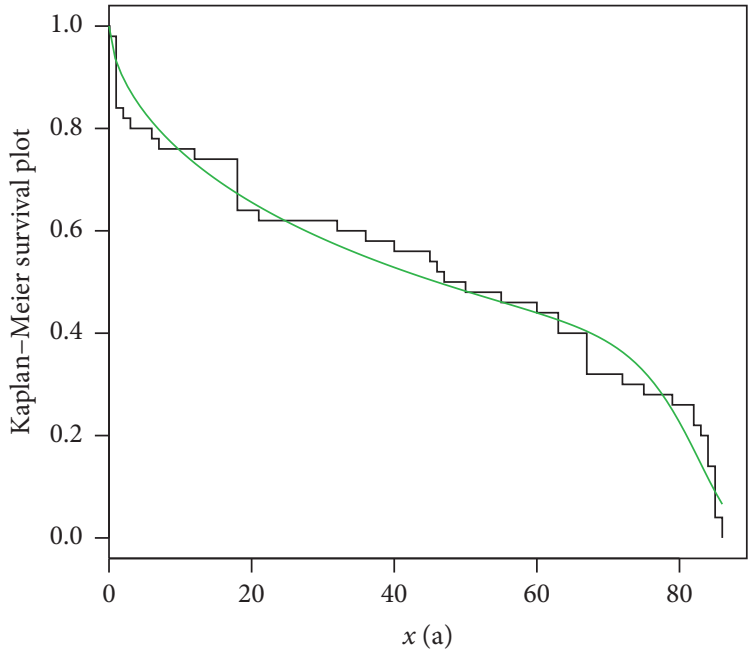

(a)

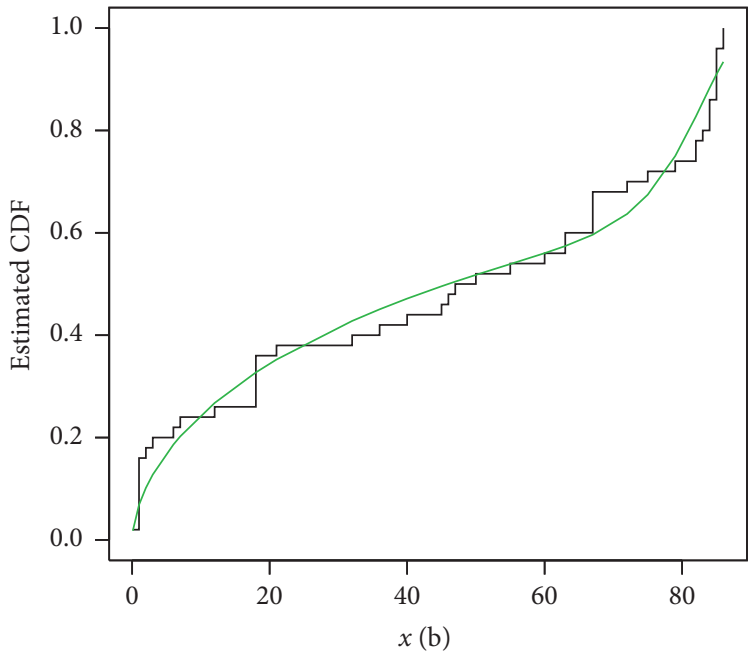

(b)

FIgURE 5: The Kaplan-Meier survival plot (a) and estimated CDF (b) of the NFEW distribution for Arset data. 


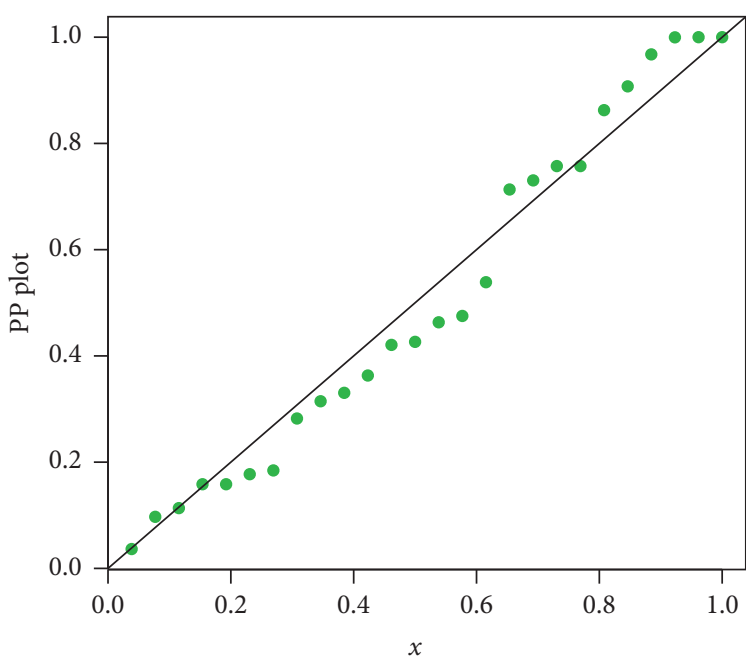

(a)

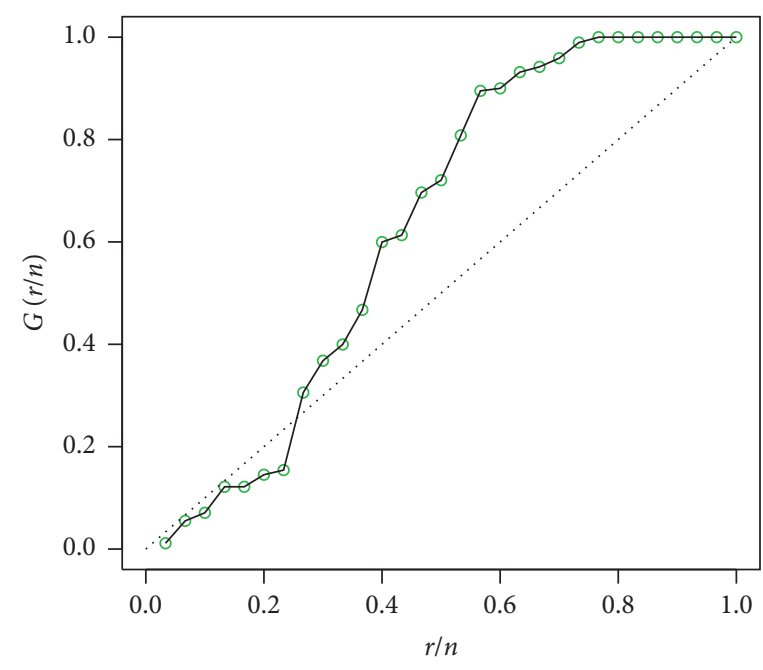

- Data 2

(b)

Figure 6: The PP-plot of the NFEW distribution and time scale TTT-transform plot for Meeker and Escobar data.

TABLE 3: MLEs with their standard errors in brackets for Meeker and Escobar data.

\begin{tabular}{lccccc}
\hline Dist. & $\hat{\beta}$ & $\hat{\alpha}$ & $\hat{\gamma}$ & $\hat{\theta}$ & $\hat{\lambda}$ \\
\hline NFEW & $0.019(0.0196)$ & $3.94(0.914)$ & & $0.372(0.095)$ & $0.973(0.263)$ \\
EW & $1.086(0.0807)$ & $0.003(0.0017)$ & & $3.076(0.2522)$ & \\
MOW & $1.013(0.2698)$ & $0.009(0.1059)$ & & $3.458(1.4248)$ & \\
NMW & $5.99 \times 10^{-8}\left(8.164 \times 10^{-8}\right)$ & $0.024(0.019)$ & $0.012(1.29)$ & $0.629(0.15)$ & $0.056(0.024)$ \\
AW & $0.019(0.018)$ & $1.320 \times 10^{-7}\left(7.435 \times 10^{-8}\right)$ & $0.604(0.197)$ & $2.830(0.974)$ & \\
APW & & $5.424 \times 10^{-12}\left(8.062 \times 10^{-8}\right)$ & $0.088(0.002)$ & $0.011(0.009)$ & $0.807(0.171)$ \\
\hline
\end{tabular}

TABLE 4: Summary values of the models fitted to Meeker and Escobar data.

\begin{tabular}{lcccc}
\hline Dist. & AIC & BIC & KS & $p$ value \\
\hline NFEW & 341.09 & 347.76 & 0.131 & 0.876 \\
EW & 375.49 & 379.70 & 0.224 & 0.104 \\
MOW & 371.93 & 376.13 & 0.223 & 0.148 \\
NMW & 344.49 & 351.46 & 0.191 & 0.482 \\
AW & 364.28 & 369.83 & 0.134 & 0.197 \\
APW & 343.82 & 349.42 & & 0.655 \\
\hline
\end{tabular}

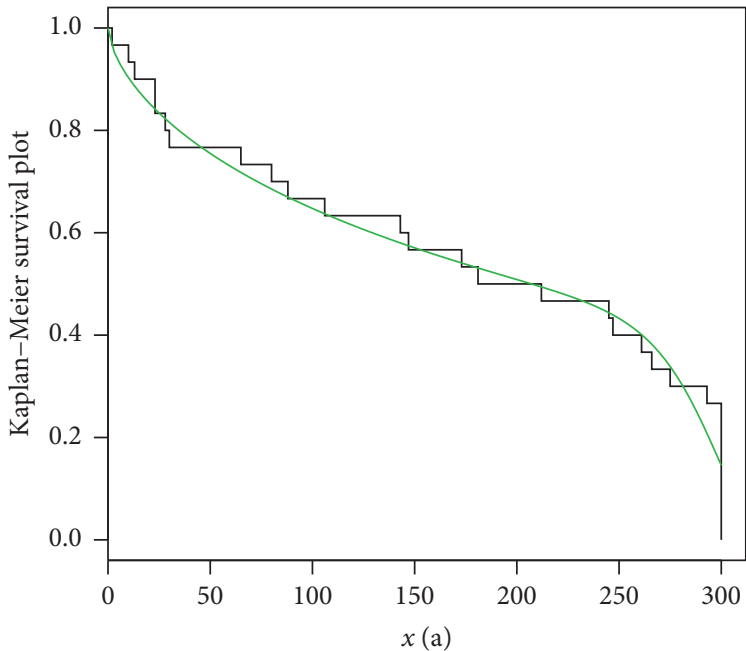

(a)

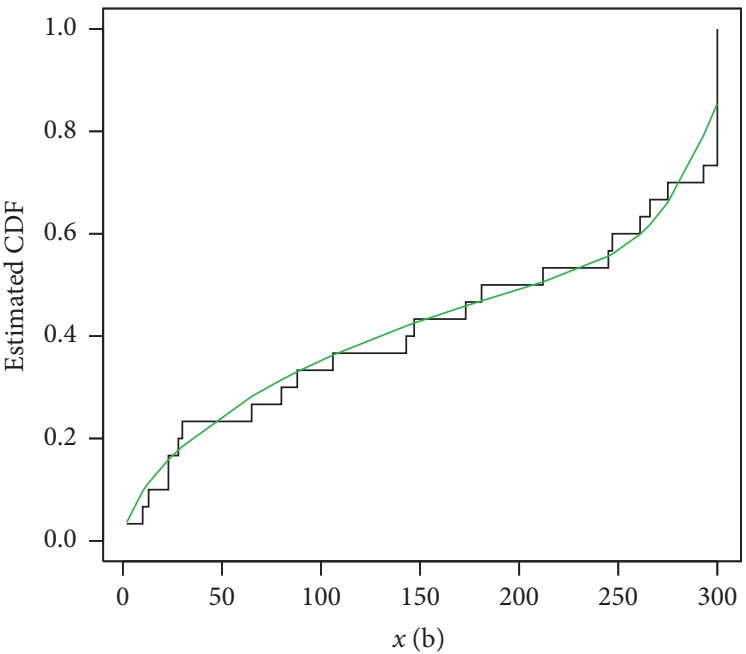

(b)

Figure 7: The Kaplan-Meier survival plot (a) and estimated CDF (b) of the NFEW distribution for Meeker and Escobar data. 


\section{Concluding Remarks}

In this study, a new flexible extended Weibull distribution with a nonmonotone hazard rate function is proposed and investigated by taking into account a convex combination of the logarithms of two cumulative hazard functions. The resulting hazard rate function of the proposed model is capable of accommodating different shapes of the failure rates including bathtub shape to describe the failure behavior of a variety of real lifetime data sets. Some mathematical properties of the proposed model have been studied. Three different characterizations of the proposed distribution are presented. The estimates of the model parameters are obtained via the method of maximum likelihood estimation. Finally, two real data sets with a bathtub-shaped failure rate, as indicated by the scaled TTTtransform plots, have been analyzed for illustrative purposes. For these data sets, some goodness of fit measures along with the $p$ values are calculated to compare the goodness of fit of the proposed model to the other competing distributions. These measures reveal that the proposed distribution provides the best fit to these bathtub-shaped data than the other distributions considered. To support the numerical results, empirical CDF and Kaplan-Meier plots are also sketched which show that the CDF of the NFEW model fits the data well, and its survival function follows the Kaplan-Meier estimate very closely. We hope that the proposed model will attract wider applications in the reliability engineering and other related fields.

\section{Appendix}

Theorem 1. Let $(\Omega, F, P)$ be a given probability space and let $H=[d, e]$ be an interval for some $d<e(d=-\infty, e=$ $\infty$ might as well be allowed). Let $X: \Omega \longrightarrow H$ be a continuous random variable with the distribution function $F(x)$ and let $q_{1}(x)$ and $q_{2}(x)$ be two real functions defined on $H$ such that

$$
E\left(q_{2}(X) \mid X \geq x\right)=E\left(q_{1}(X) \mid X \geq x\right) \eta(x), \quad x \in H,
$$

is defined with some real function $\eta(x)$. Assume that $q_{1}(x), q_{2}(x) \in C^{1}(H), \eta(x) \in C^{2}(x)$, and $F(x)$ is twice continuously differentiable and strictly monotone function on the set $H$. Finally, assume that the equation $\eta(x) q_{1}(x)=$ $q_{2}(x)$ has no real solution in the interior of $H$. Then, $F(x)$ is uniquely determined by the functions $q_{1}(x), q_{2}(x)$, and $\eta(x)$, particularly,

$$
F(x)=\int_{a}^{x} C\left|\frac{\eta^{\prime}(u)}{\eta(u) q_{1}(u)-q_{2}(u)}\right| \exp (-s(u)) \mathrm{d} u,
$$

where the function $s(u)$ is a solution of the differential equation $s^{\prime}(u)=\left(\eta^{\prime}(u) q_{1}(u) /\left(\eta(u) q_{1}(u)-q_{2}(u)\right)\right)$ and $C$ is the normalization constant, such that $\int_{H} d F=1$.

\section{Data Availability}

The data set is provided in the manuscript.

\section{Conflicts of Interest}

The authors declare that there are no conflicts of interest regarding the publication of this paper.

\section{Acknowledgments}

The first author acknowledges the support of the National Social Science Foundation of China (no. 17BZZ006), Funds of Tianjin Philosophy and Social Science Foundation of China (no. TJGL17-023), and Cultivation Funds for National Project of Tianjin University of Commerce (no. 161137). The authors also acknowledge Dr. Saima K. Khosa for helping in revision.

\section{References}

[1] G. S. Mudholkar and D. K. Srivastava, "Exponentiated Weibull family for analyzing bathtub failure-rate data," IEEE Transactions on Reliability, vol. 42, no. 2, pp. 299-302, 1993.

[2] M. Xie, Y. Tang, and T. N. Goh, "A modified Weibull extension with bathtub-shaped failure rate function," Reliability Engineering \& System Safety, vol. 76, no. 3, pp. 279-285, 2002.

[3] T. Zhang and M. Xie, "On the upper truncated Weibull distribution and its reliability implications," Reliability Engineering \& System Safety, vol. 96, no. 1, pp. 194-200, 2011.

[4] M. Bebbington, C.-D. Lai, and R. Zitikis, "A flexible Weibull extension,” Reliability Engineering \& System Safety, vol. 92, no. 6, pp. 719-726, 2007.

[5] Z. Ahmad, "The Zubair-G family of distributions: properties and applications," Annals of Data Science, vol. 5, pp. 1-14, 2018.

[6] Z. Ahmad, M. Ilyas, and G. G. Hamedani, "The extended alpha power transformed family of distributions: properties and applications," Journal of Data Science, vol. 17, no. 4, pp. 726-741, 2019.

[7] Z. Ahmad, M. Elgarhy, and N. Abbas, "A new extended alpha power transformed family of distributions: properties and applications," Journal of Statistical Modelling: Theory and Applications, vol. 1, no. 1, pp. 13-28, 2019.

[8] C. D. Lai, M. Xie, and D. N. P. Murthy, "A modified Weibull distribution," IEEE Transactions on Reliability, vol. 52, no. 1, pp. 33-37, 2003.

[9] G. O. Silva, E. M. M. Ortega, and G. M. Cordeiro, "The beta modified Weibull distribution," Lifetime Data Analysis, vol. 16, no. 3, pp. 409-430, 2010.

[10] S. K. Upadhyay and A. Gupta, "A Bayes analysis of modified Weibull distribution via Markov chain Monte Carlo simulation," Journal of Statistical Computation and Simulation, vol. 80, no. 3, pp. 241-254, 2010.

[11] A. A. Soliman, A. H. Abd-Ellah, N. A. Abou-Elheggag, and E. A. Ahmed, "Modified Weibull model: a Bayes study using MCMC approach based on progressive censoring data," Reliability Engineering \& System Safety, vol. 100, pp. 48-57, 2012.

[12] M. Xie and C. D. Lai, "Reliability analysis using an additive Weibull model with bathtub-shaped failure rate function," Reliability Engineering \& System Safety, vol. 52, no. 1, pp. 87-93, 1996.

[13] S. J. Almalki and J. Yuan, "A new modified Weibull distribution," Reliability Engineering \& System Safety, vol. 111, pp. 164-170, 2013. 
[14] B. Singh, "An additive perks-Weibull model with bathtubshaped hazard rate function," Communications in Mathematics and Statistics, vol. 4, no. 4, pp. 473-493, 2016.

[15] A. M. Sarhan and M. Zaindin, "Modified Weibull distribution," Applied Sciences, vol. 11, pp. 123-136, 2009.

[16] A. J. Lemonte, G. M. Cordeiro, and E. M. M. Ortega, "On the additive Weibull distribution," Communications in Statistics-Theory and Methods, vol. 43, no. 10-12, pp. 2066-2080, 2014.

[17] S. Nadarajah and S. Kotz, "On some recent modifications of Weibull distribution," IEEE Transactions on Reliability, vol. 54, no. 4, pp. 561-562, 2005.

[18] W. Glänzel, "A characterization theorem based on truncated moments and its application to some distribution families," in Mathematical Statistics and Probability Theory, pp. 75-84, Springer, Berlin, Germany, 1987.

[19] W. Glanzel, "Some consequences of a characterization theorem based on truncated moments," Statistics, vol. 21, no. 4, pp. 613-618, 1990.

[20] G. G. Hamedani, On Certain Generalized Gamma Convolution Distributions II, Marquette University, Milwaukee, WI, USA, 2013.

[21] A. Marshall and I. Olkin, "A new method for adding a parameter to a family of distributions with application to the exponential and Weibull families," Biometrika, vol. 84, no. 3, pp. 641-652, 1997.

[22] M. V. Aarset, "How to identify a bathtub hazard rate," IEEE Transactions on Reliability, vol. R-36, no. 1, pp. 106-108, 1987.

[23] W. Q. Meeker and L. A. Escobar, Statistical Methods for Reliability Data, John Wiley \& Sons, Hoboken, NJ, USA, 2014. 